\title{
A Simple Extension of Digital Filter-based Turbulent Inflow to Non-Uniform Structured Grids
}

\author{
N. S. Dhamankar ${ }^{a, *}$, G. A. Blaisdell ${ }^{a}$, and A. S. Lyrintzis ${ }^{b}$ \\ ${ }^{a}$ School of Aeronautics and Astronautics, Purdue University, West Lafayette, IN-47907 \\ ${ }^{b}$ Embry-Riddle Aeronautical University, Daytona Beach, FL-32114
}

June 8, 2016

\begin{abstract}
The digital filter-based turbulence generator is one of the widely used synthetic turbulence inflow boundary conditions for large-scale-resolving flow simulations. However, the requirement of using uniformly-spaced Cartesian meshes and the limitation of enforcing a constant integral length scale along a given direction restrict the applicability of the method severely. The current paper describes an easy-to-implement and computationally inexpensive approach to extend the digital filter-based inflow to non-uniform structured meshes, using a mapping between the physical curvilinear mesh and a computational uniformly-spaced Cartesian mesh. The method also allows a limited control on the variation of integral length scales over the inflow plane. A zero-pressure-gradient, flat plate turbulent boundary layer is simulated using the proposed method and the distance required by the generated fluctuations to adapt into realistic turbulence is estimated to be about 10 boundary layer thicknesses. The method was developed for and has been used in simulations of turbulent boundary layers along nozzle walls for jet noise applications.
\end{abstract}

Keywords - turbulent inflow boundary condition, digital filter, curvilinear structured grids, large eddy simulation, turbulent boundary layer

\section{Digital Filter-based Turbulent Inflow}

For synthetic turbulence inflow boundary conditions, it is both essential and challenging to introduce realistic spatial coherence in the fluctuating inflow quantities. Not prescribing any energy carrying large length scales at the inflow results in an immediate annihilation of such synthetic fluctuations within the flow domain [1]. One effective way of prescribing desired length scales in a random signal is through application of properly designed digital filters [2]. This method has become one of the widely used synthetic turbulence boundary conditions in the last decade (see [3] for a recent review) and a short description of its formulation is provided in this section.

For illustration purposes, consider a 2-D computational domain having a 1-D line discretized in $N-1$ intervals as the inlet. A random number $r$, picked from a normal distribution with unit variance, is assigned to each of the $N$ grid points. The filtering operation generates correlated length scales in this random signal. A 1-D filter function is given by

$$
\grave{u}_{k}=\sum_{a=-W}^{W} b_{a} r_{k+a},
$$

where $b_{a}$ are the filter coefficients and $W$ is the filter stencil half-width. $\grave{u}_{k}$ represents the filtered velocity fluctuation for a particular velocity component $u$ at the location indexed $k$. It can be shown that the

*Corresponding author. Email: nsdhamankar@gmail.com 
two-point correlation function for this filtered velocity component takes the following form [2]:

$$
R_{\grave{u} \grave{u}}(p \Delta s)=\frac{\overline{\grave{u}_{k} \grave{u}_{k+p}}}{{\grave{u_{k} \grave{u}_{k}}}_{n}}=\left(\sum_{a=-W+p}^{W} b_{a} b_{a-p}\right) /\left(\sum_{a=-W}^{W} b_{a}^{2}\right),
$$

where $(p \Delta s)$ is the separation in terms of $p$ intervals of uniform grid spacing $\Delta s$. The overbar indicates an average over all points on the 1-D inlet line, from $k=1$ to $k=N$. Note that Equation (2) does not use index notation and $k$ is simply the location index of a grid point. The filter has to be designed in such a way that the correlation established by Equation (2) matches in form with the correlation observed in the physical flow to be simulated, thus prescribing a realistic integral length scale in the $\grave{u}$ fluctuations. The details of the filter design, extension to 3-D domains, and additional processing to enforce a desired Reynolds stress tensor are not included in this paper for the sake of brevity. A complete description of the procedure used by the authors can be found in [4].

\section{Extension to Non-Uniform Grids}

The filtering operation described in section 1 is limited to uniformly-spaced Cartesian grids and cannot be readily applied to inlet planes in curvilinear grids, which is highly desirable for most practical applications. For example, in large eddy simulations performed to study the noise produced by turbulent jets issuing from different nozzle designs, a realistic turbulent boundary layer is desired on the inner nozzle walls. This turbulent boundary layer gives rise to a turbulent shear layer in the free jet and is crucial for the downstream flow dynamics and the associated noise generation. The most common nozzle cross-section shape is circular and a cylindrical grid provides optimum resolution of the initial jet shear layer. However, the digital filter-based inflow cannot be applied in such a cylindrical grid. Another shortcoming of the digital filter-based technique is the limitation of using a constant integral length scale in a particular direction. This is especially important in wall-bounded flows where the integral length scale is supposed to reduce to zero towards the wall.

For use in curvilinear grids, the usually recommended procedure $[5,6]$ is to generate a separate uniformly-spaced Cartesian grid larger than or equal in extent to the actual curvilinear inlet grid. The filtering operation is first performed on the uniform grid and then interpolation is used to transfer the fluctuations to the actual inlet. This method involves additional computational effort for interpolation, as well as complicates the implementation, especially for parallel solvers.

To vary the integral length scale over the inlet plane, one approach is to vary the filter coefficients spatially, although a strong variation through change of the filter coefficients can result in deviation of the correlation function from its prescribed shape [2]. As an alternative, a zonal approach has been proposed by Veloudis et al. [7] to allow integral length scales to vary over the inflow plane. In their approach, the inlet plane is split into several uniformly-spaced Cartesian grids. The filtering operation is performed in each zone separately with different filter coefficients, thus resulting in different integral length scales in separate zones. A stepwise variation in length scales is possible with this approach, although it suffers from discontinuities in fluctuations at the zone boundaries. Also, in general curvilinear grids, the two-step process of generating fluctuations on several uniform zones and then interpolating the fluctuations onto the actual inlet is both complicated to implement in parallel and requires more computational effort. In certain curvilinear geometries, such a zonal approach cannot be readily applied to have a desired length scale variation over the inlet plane. For example, in a pipe flow simulation, the length scales are required to decrease radially towards the wall. A Cartesian zone-based approach cannot handle such requirements.

In the current work, a different way of introducing the digital filter-based fluctuations in curvilinear grids is suggested. This approach does not involve the excessive computational cost of interpolation and allows smoother variation in length scales, albeit with some limitations. When the governing equations are solved on a structured curvilinear grid with finite-difference discretization, the physical mesh is mapped onto a uniformly-spaced Cartesian computational mesh. The filtering operation can be performed on such a mapped mesh which is uniformly-spaced by definition. Parallelizing this operation is straightforward compared to the implementation of interpolation-based methods. The required length scales are specified in terms of intervals on this mapped grid. After filtering establishes these length scales, the resulting velocity fluctuations are simply mapped back onto the physical grid. If a smooth grid stretching is employed on the physical grid, it causes the imposed length scales to vary in an equivalent smooth manner. 


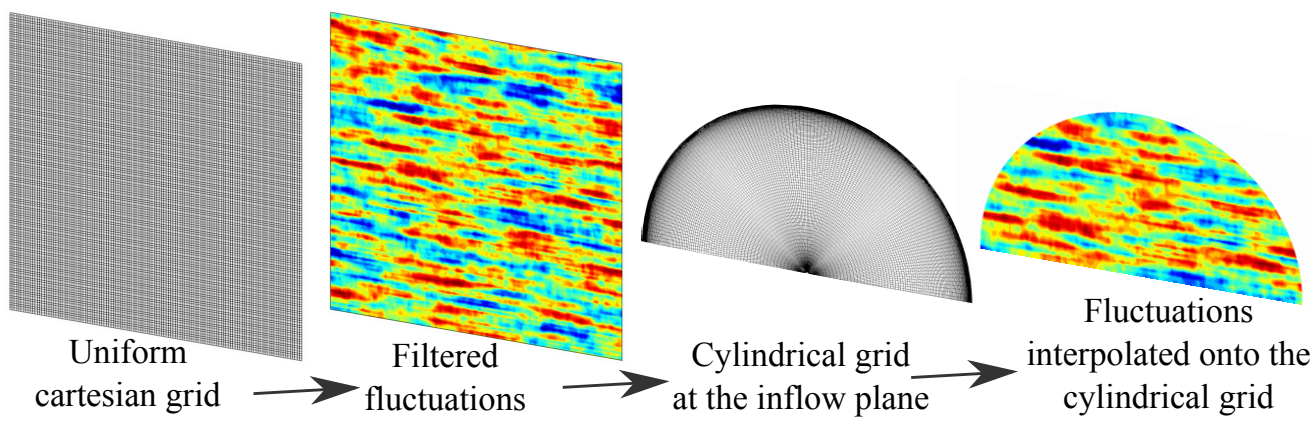

(a) Interpolation-based approach.

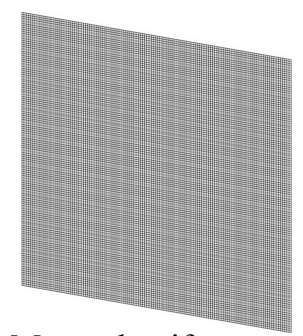

Mapped uniform cartesian grid

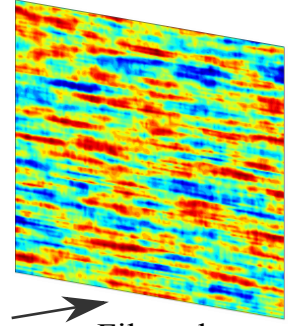

Filtered

fluctuations

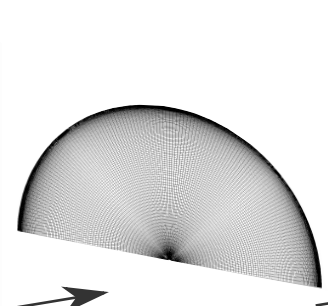

Cylindrical grid at the inflow plane

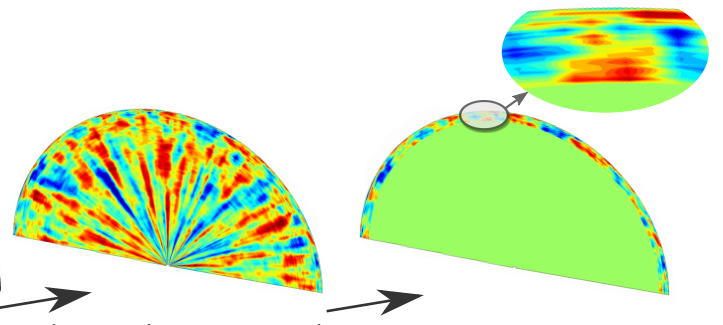

Fluctuations mapped Fluctuations limited only onto the cylindrical grid within the boundary layer

(b) Mapping-based approach.

Figure 1: Different approaches for extension of the digital filter-based turbulent inflow to curvilinear structured grids (reproduced from [3]).

The difference between the current approach and the interpolation-based approach is demonstrated in Fig. 1, for a case where turbulent fluctuations are required on a 2-D cylindrical grid, such as a pipe flow simulation. It is clear from Fig. 1(a) that the interpolation-based approach is not capable of enforcing the correct orientation of the length scales on the cylindrical mesh. The grid is generally designed to be finer closer to a wall to resolve the boundary layer, and therefore the mapping-based approach will inherently produce decreasing length scales towards a wall. This desirable property is seen in Fig. 1(b) in the radial direction towards the outer wall boundary. However, mapping produces undesirable results when the grid density does not directly correspond to the integral length scales in a region. This effect can also be observed in Fig. 1(b). Since the azimuthal grid spacing does not decrease towards the outer wall, the azimuthal length scale can not be decreased towards the wall using the mapping approach. Also, structures highly stretched in the radial direction occur near the centerline due to the very small azimuthal spacing in that region. However, in multiple applications, it would be possible and acceptable to avoid such artifacts by limiting the filtering operation and the fluctuations only to a region belonging to the turbulent boundary layer. For example, for an inlet to a circular nozzle, turbulent fluctuations can be added only in the boundary layer near the outer wall as shown in Fig. 1(b) when the core of the flow is expected to have negligible turbulence. Although the resulting fluctuations represent the physical length scales in only an approximate manner, they are sufficient to trigger the development of realistic turbulence downstream of the inflow plane, as demonstrated in section 3.

\section{Testing and Results}

The proposed modification to the digital filter-based turbulent inflow is tested using a stretched Cartesian mesh for a flat plate, zero-pressure-gradient, turbulent boundary layer (ZPGTBL). Most synthetic turbulence generators (such as the currently used method) require a nonzero adaptation distance downstream of the inflow plane to recover from modeling errors and establish realistic fluctuations [3]. Due to the availability of semi-empirical theoretical relations and direct numerical simulation (DNS) databases, a 
ZPGTBL provides a useful test case to evaluate the adaptation distance required for any turbulent inflow method. A low freestream Mach number is used in the current test $\left(M_{\infty}=0.2\right)$ to allow comparison with the incompressible ZPGTBL simulation performed by Lund et al. [1] using the recycling-rescaling-based (RR) turbulent inflow method. In the RR method, the velocity fluctuations from a downstream location are rescaled and reintroduced at the domain inlet. The $R R$ method has been shown to not require an adaptation distance and to produce a turbulent boundary layer that follows the canonical behavior. Therefore, it is an appropriate reference turbulent inflow method to assess the effectiveness of the current method. It is to be noted that although the $\mathrm{RR}$ method produces excellent inflow conditions for ZPGTBLs, it suffers from certain limitations such as the requirement of a concurrent inflow-generating simulation (or a considerable separation between the inflow plane and the downstream sampling plane even if the recycling box is embedded within the main simulation), the necessity of boundary layer scaling laws to hold near the inflow plane, the possibility of introducing spurious low-frequency modes in the flow-field, and a relatively complicated implementation, especially for parallel solvers [3]. Many synthetic turbulence generators such as the currently presented method are in general more flexible and suitable for applications of practical interest.

The current test is performed using an implicit large eddy simulation approach. For details of the governing equations and numerical methods, please refer to the previous work by the authors [4]. The boundary layer thickness $\left(\delta_{99}\right)$ is defined to be the wall-normal location where the mean streamwise velocity is $99 \%$ of the freestream velocity $\left(U_{\infty}\right)$, and its value at the domain inlet $\left(\delta_{99, i}\right)$ is considered to be the reference length scale for the simulation. The grid extents are $L_{x}$ (streamwise) $\times L_{y}$ (wall-normal) $\times$ $L_{z}($ spanwise $)=[50 \times 15 \times \pi / 2] \delta_{99, i}$. The longer axial domain extent is necessary to examine the boundary layer evolution over a sufficiently long distance beyond the initial adaptation zone near the inflow boundary. The number of grid points in the three directions are $N_{x} \times N_{y} \times N_{z}=1,344 \times 160 \times 128$. The Reynolds number based on freestream quantities and boundary layer momentum thickness $(\theta)$ spans a range of $R e_{\theta} \approx 1,400$ to 2,250 through the domain length. The current inflow generation method requires inputs for the desired mean velocity profile, Reynolds stress profiles and integral length scales at the inlet. For the mean streamwise velocity profile, an expression for the incompressible law of the wall, comprised of an expression for the viscous sublayer and the log layer by Reichardt [8], and a wake portion consisting of the law of the wake by Coles [9] is used. It is given by

$$
u^{+}=\frac{\ln \left(1+0.4 y^{+}\right)}{\kappa}+7.23486\left(1-e^{-y^{+} / 11}-\left(y^{+} / 11\right) e^{-0.33 y^{+}}\right)+\frac{2 \Pi}{\kappa} \sin ^{2}\left(\frac{\pi}{2} \eta\right),
$$

with $\eta=y / \delta_{99}, \kappa=0.41$, and $\Pi=0.5$. Note that in Equation (3), coefficients from the original expression by Reichardt [8] are modified to better match the log-law section with the expression $u^{+}=$ $\log \left(y^{+}\right) / 0.41+5$, which is usually employed for incompressible ZPGTBLs. Based on the friction velocity at the domain inlet $\left(u_{\tau, i}\right)$, the uniform streamwise and spanwise grid spacings are $\Delta x^{+}=18.9$ and $\Delta z^{+}=6.3$, respectively. In the wall-normal direction, the grid is stretched away from the wall. The minimum wall-normal spacing at the wall is $\Delta y_{\min }^{+}=0.75$, and at the edge of the boundary layer at the inlet (at $y=\delta_{99, i}$ ), it is $\Delta y^{+}=13.2$. Note that in the simulation by Lund et al. [1], the corresponding spacings were $\Delta x^{+} \approx 64, \Delta z^{+} \approx 15$, and $\Delta y_{\min }^{+}=1.2$. Therefore, the currently used grid is deemed to provide sufficient resolution. The reference turbulence intensities and Reynolds shear stress profile are taken from a DNS of a ZPGTBL at $R e_{\theta}=1,410$ by Spalart [10]. In the previous tests with the current method [4], it has been observed that the downstream flow evolution is relatively independent of the length scales prescribed at the inlet, as long as the Reynolds number is large enough for the flow to be fully turbulent and the prescribed length scales are at least approximately close to the integral length scales in the corresponding physical flow. In the current test, the streamwise and spanwise length scales are chosen to be $1.28 \delta_{99, i}$ and $0.25 \delta_{99, i}$, respectively. In the wall-normal direction, the length scale is chosen to be about $0.25 \delta_{99, i}$ in the middle of the boundary layer. Due to grid stretching away from the wall, the wall-normal length scale decreases towards the wall, and increases away from the wall. The spanwise and wall-normal length scales are prescribed using the filtering approach described in section 2. The streamwise length scales are prescribed using a cost-effective approach suggested by Xie and Castro [6] that correlates fluctuations at a given timestep with those from the previous timestep incorporating a specified time scale of fluctuations.

An adiabatic viscous wall boundary condition [11] is used on the wall at the bottom $(y=0)$ plane. At the top face of the domain, a stress-free symmetry boundary condition [4] is applied. A characteristicbased outflow condition [12] with viscous corrections [13] is applied at the outflow plane. In the spanwise 
direction, periodicity is assumed. The mean profiles enforced at the inlet for all primitive variables are used as initial conditions throughout the domain. The solution is time-advanced for a total of $1.26 \times 10^{6}$ steps with a timestep of $\triangle t=2.8 \times 10^{-4} \delta_{99, i} / U_{\infty}$, which restricts the maximum Courant-Friedrichs-Lewy (CFL) number to be about 0.98 and provides a stable solution with the explicit fourth-order Runge-Kutta time advancement scheme used in the present study. The total simulation time is $352.8 \delta_{99, i} / U_{\infty}$. The initial $100.8 \delta_{99, i} / U_{\infty}$ worth of simulation time is alloted to the removal of transients resulting from unphysical initial conditions. The statistical averaging interval corresponds to the latter $252 \delta_{99, i} / U_{\infty}$ worth of simulation time.

The results of the simulation are now discussed. All the quantities presented here are both timeaveraged and additionally averaged along the statistically homogeneous spanwise direction. Fig. 2 shows the streamwise evolution of four boundary layer parameters, and compares the current results with those using the RR method, as well as with estimates from a momentum integral analysis performed following the procedure outlined by Lund et al. [1] and using Equation (3) as the reference velocity profile. For computing boundary layer integral quantities, such as $\theta$ and the shape factor $(H)$, the necessary integration has been carried out between the wall and a height of $3 \delta_{99}$ at each streamwise location. In Figs. 2(a) to 2(d), the relevant comparison is between the slopes of the evolution curves and not between their absolute values, since offsets are introduced due to having non-identical velocity profiles at the starting location. Fig. 2(a) shows the momentum thickness $(\theta)$ evolution. It is seen that $d \theta / d x$ is negative very close to the inflow plane at $x=0$ in the current results. This unphysical behavior is due to the required adaptation distance, within which artificially generated fluctuations trigger the development of sustained realistic turbulence. The location where $d \theta / d x$ reaches a value that agrees well with the momentum integral estimate can be considered to provide one quantitative measure of the adaptation distance. In the current results, $d \theta / d x$ turns positive at about $x=1 \delta_{99, i}$, and achieves a value within $11 \%$ of the momentum integral estimate by $x=10 \delta_{99, i}$. Considering the small magnitude of $d \theta / d x$, it can be said that an adaptation distance of about $10 \delta_{99, i}$ is sufficient for practical applications of the method. The rest of the evolution plots in Fig. 2 use $R e_{\theta}$ as the abscissa, and therefore, the initial decrease in $\theta$ exhibits itself in the form of artifacts (multiple values for a single value of $R e_{\theta}$ ) at the beginning of the curves for the current results. Fig. 2(b) shows that the boundary layer thickness exhibits reasonably correct growth beyond $R e_{\theta}=1,500$, which corresponds to about $x=8.5 \delta_{99, i}$. Fig. 2(c) plots the skin friction coefficient $\left(C_{f}\right)$ as a function of $R e_{\theta}$. It is seen that within the adaptation distance, $C_{f}$ initially drops, then recovers and follows a reasonable evolution beyond $R_{\theta}=1,500$ (corresponding to $\left.x=8.5 \delta_{99, i}\right)$. A similar trend is seen in Fig. $2(\mathrm{~d})$ for the shape factor. Thus, based on the evolution characteristics of all the properties presented in Fig. 2, the adaptation distance can be estimated to be about $10 \delta_{99, i}$. It is to be noted that even using the zonal approach [7], the digital filter-based inflow has been shown to require about 10 boundary layer thicknesses to recover a reasonable value of $C_{f}$ [14]. Therefore, the current approach requires an expectedly long adaptation distance, while being simpler to implement.

Turbulence statistics near $x=10 \delta_{99, i}$ are now examined. Fig. 3(a) plots the mean streamwise velocity profile in inner viscous units and Fig. 3(b) plots the root mean square (r.m.s.) fluctuating velocities and the Reynolds shear stress at a streamwise location corresponding to $R e_{\theta}=1,530$. This location is at $x=9.94 \delta_{99, i}$ in the current test. It is seen that the current velocity profile matches well with that from the RR method, and both show similar deviation from the profile given by Equation (3) excluding the wake portion. The differences towards the outer edge of the profiles are due to the different $C_{f}$ values for the simulation using the RR method and the current results. Fig. 3(b) shows that all the Reynolds stresses have recovered and agree well with the estimates from the RR method. Thus, an adaptation distance of about $10 \delta_{99, i}$ is sufficient for establishment of reasonably correct turbulence statistics as well.

\section{Summary}

This paper describes a simple and cost-effective method to extend digital filter-based turbulent inflow generation to non-uniform structured meshes, by performing the filtering operation on a mapped uniform computational mesh. Test results indicate that the method requires an adaptation distance of about 10 boundary layer thicknesses to establish reasonably correct behavior for evolution of boundary layer parameters, mean velocity profile, and turbulence statistics. This method has been successfully used with a curvilinear structured grid to study the noise generated by an overexpanded turbulent jet from a 


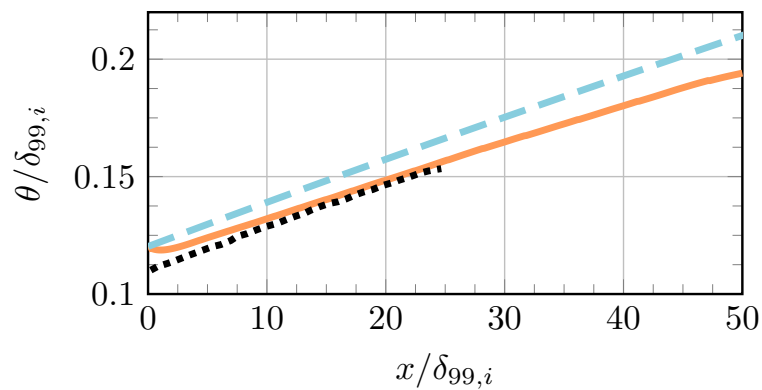

(a) Evolution of momentum thickness.

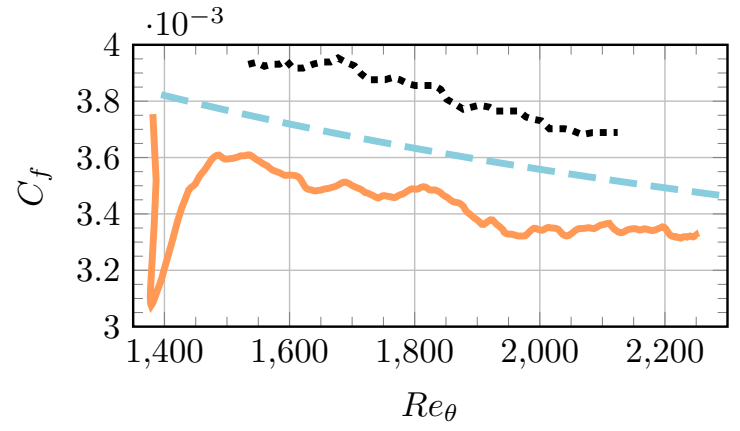

(c) Evolution of skin friction coefficient.

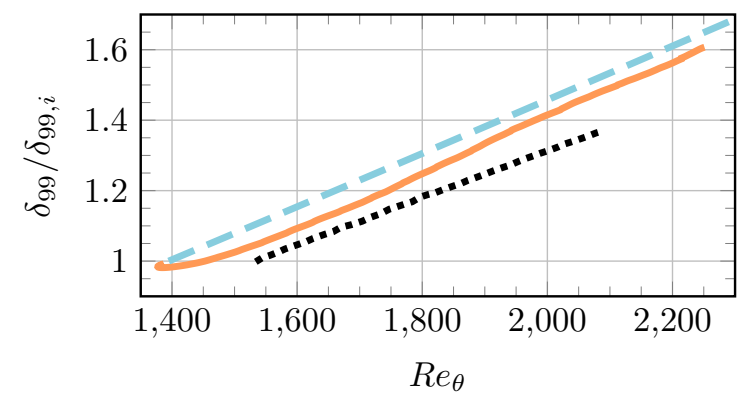

(b) Evolution of boundary layer thickness.

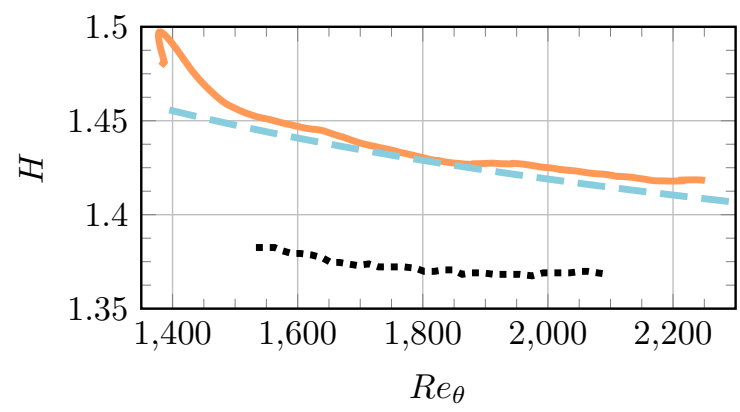

(d) Evolution of shape factor.

Figure 2: Comparison of streamwise evolution of boundary layer parameters. Current method $\longrightarrow$, momentum integral estimate $-=$, RR method [1] $\cdots \cdot \cdot$.

converging-diverging beveled nozzle [15].

\section{Acknowledgments}

We sincerely thank Chandra Martha and Yingchong Situ whose prior implementation of a large eddy simulation code was used as the basis for the current work. This material is based upon work supported by the National Science Foundation (NSF) under grant number OCI-0904675. The Stampede cluster of the Texas Advanced Computing Center (TACC) was utilized for the presented simulation under allocation TG-ASC040044N.

\section{References}

[1] T. Lund, X. Wu, and K. Squires. Generation of turbulent inflow data for spatially-developing boundary layer simulations. Journal of Computational Physics, 140(2):233-258, 1998.

[2] M. Klein, A. Sadiki, and J. Janicka. A digital filter based generation of inflow data for spatially developing direct numerical or large eddy simulations. Journal of Computational Physics, 186(2):652-665, 2003.

[3] N. Dhamankar, G. Blaisdell, and A. Lyrintzis. An overview of turbulent inflow boundary conditions for large eddy simulations (invited). Presented at the 22nd AIAA Computational Fluid Dynamics Conference, AIAA Paper 2015-3213, 2015.

[4] N. Dhamankar, C. Martha, Y. Situ, K. Aikens, G. Blaisdell, A. Lyrintzis, and Z. Li. Digital filterbased turbulent inflow generation for jet aeroacoustics on non-uniform structured grids. Presented at the 52nd AIAA Aerospace Sciences Meeting, AIAA Paper 2014-1401, 2014. 


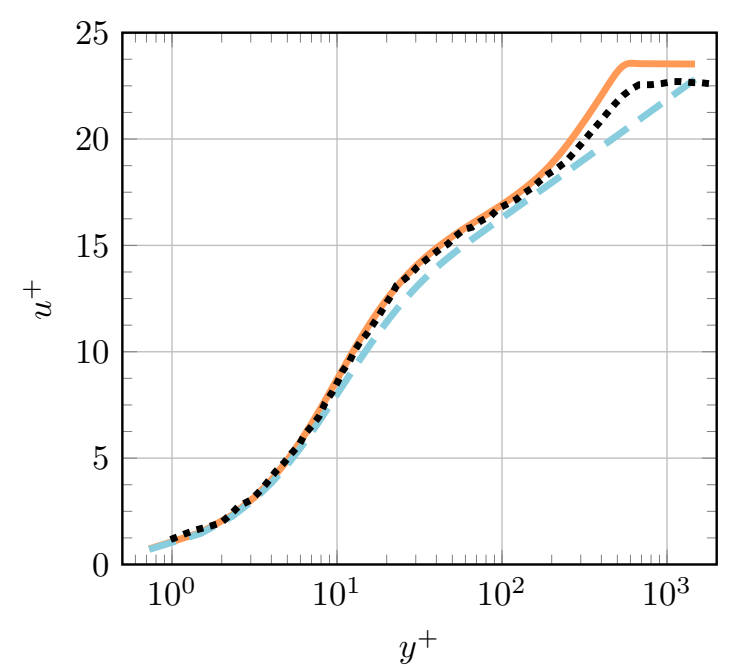

(a) Mean streamwise velocity profile in inner-scaled variables.

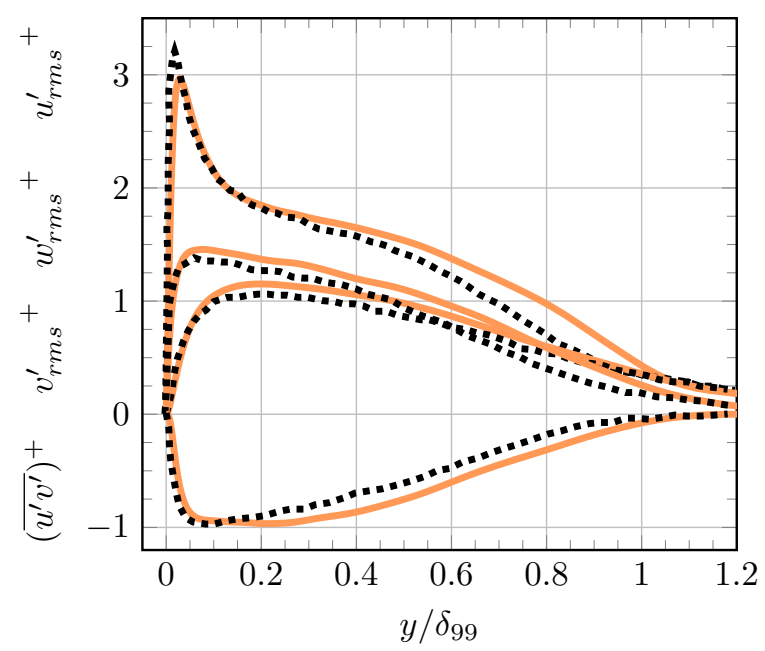

(b) r.m.s. fluctuating velocities (scaled by $u_{\tau}$ ) and Reynolds shear stress (scaled by $u_{\tau}^{2}$ ).

Figure 3: Comparison of turbulence statistics at a streamwise location corresponding to $R e_{\theta}=1,530$. Current method $=$, Equation $(3)($ with $\Pi=0)=-$, RR method [1] $\cdots \cdots \cdot$

[5] Z. Rana, B. Thornber, and D. Drikakis. On the importance of generating accurate turbulent boundary condition for unsteady simulations. Journal of Turbulence, 12(35):1-39, 2011.

[6] Z. Xie and I. Castro. Efficient generation of inflow conditions for large eddy simulation of street-scale flows. Flow Turbulence and Combustion, 81:449-470, 2008.

[7] I. Veloudis, Z. Yang, J. McGuirk, G. Page, and A. Spencer. Novel implementation and assessment of a digital filter based approach for the generation of LES inlet conditions. Flow Turbulence and Combustion, 79:1-24, 2007.

[8] H. Reichardt. Vollständige darstellung der turbulenten geschwindigkeitsverteilung in glatten leitungen. Z. Angew. Math. Mech., 31:208-219, 1951.

[9] D. Coles. The law of the wake in the turbulent boundary layer. Journal of Fluid Mechanics, 1:191-226, 1956.

[10] P. Spalart. Direct simulation of a turbulent boundary layer up to $\operatorname{Re}_{\theta}=1410$. Journal of Fluid Mechanics, 187:61-98, 1988.

[11] N. Dhamankar. Boundary conditions towards realistic simulation of jet engine noise. Master's thesis, School of Aeronautics and Astronautics, Purdue University, West Lafayette, IN, USA, December 2012 .

[12] J. Kim and D. Lee. Generalized characteristic boundary conditions for computational aeroacoustics. AIAA Journal, 38(11):2040-2049, 2000.

[13] G. Lodato, P. Domingo, and L. Vervisch. Three-dimensional boundary conditions for direct and large-eddy simulation of compressible viscous flows. Journal of Computational Physics, 227(10):5105-5143, 2008.

[14] E. Touber and N. Sandham. Large-eddy simulation of low-frequency unsteadiness in a turbulent shock-induced separation bubble. Theoretical and Computational Fluid Dynamics, 23:79-107, 2009.

[15] K. Aikens, G. Blaisdell, A. Lyrintzis, and Z. Li. Analysis of converging-diverging beveled nozzle jets using large eddy simulation with a wall model. Presented at the 53rd AIAA Aerospace Sciences Meeting, AIAA Paper 2015-0509, 2015. 